\title{
1 Rats show a preference for certain unfamiliar strains of rats
}

2

3 Hiroki Kogo, Yasushi Kiyokawa, and Yukari Takeuchi

4

5 Laboratory of Veterinary Ethology, The University of Tokyo, 1-1-1 Yayoi, Bunkyo-ku,

6 Tokyo 113-8657, Japan

7

8

9 Corresponding author: Yasushi Kiyokawa, Laboratory of Veterinary Ethology,

10 The University of Tokyo, 1-1-1 Yayoi, Bunkyo-ku, Tokyo 113-8657, Japan

11 E-mail: akiyo@mail.ecc.u-tokyo.ac.jp

12 Tel.: +81-3-5841-7577; Fax: +81-3-5841-8190

13 


\section{Abstract}

15 Humans show distinct social behaviours when we recognise social similarity in

16 opponents that are members of the same social group. However, little attention has been

17 paid to the role of social similarity in non-human animals. In Wistar subject rats, the

18 presence of an unfamiliar Wistar rat mitigated stress responses, suggesting the

19 importance of social similarity in this phenomenon. We found that the presence of

20 unfamiliar Sprague-Dawley (SD) or Long-Evans (LE) rats, but not an unfamiliar

21 Fischer 344 (F344) rat, similarly mitigated stress in subject rats. It is therefore possible

22 that the subject rats recognised social similarity to unfamiliar SD and LE rats. In this

23 study, we demonstrated that Wistar subject rats were capable of categorizing unfamiliar

24 rats based on their strain, and that Wistar subjects showed a preference for unfamiliar

25 Wistar, SD, and LE rats over F344 rats. However, the subject rats did not show a

26 preference among Wistar, SD, and LE rats. In addition, the results were not due to an

27 aversion to F344 rats, and preference was not affected when anaesthetised rats were

28 presented to subject rats. The findings suggested that rats recognise social similarity to

29 certain unfamiliar strains of rats.

31 Keywords: Group identity; Group preference; Ingroup favouritism; Signature mixture

32 Social buffering 


\section{Background}

In social animals, behaviours towards conspecific individuals are influenced by

40 two independent factors. One is individual familiarity with an opponent, which is

41 assessed based on the individual identity of the opponent. The clearest examples of such

42 behaviours would include those observed exclusively between a mother and her infant

43 or between pair-bonded mates [1,2]. Behaviours towards conspecific individuals other

44 than mothers or mates are also affected by individual familiarity; for example, animals

45 are prone to exhibit pro-social behaviour towards familiar individuals [3], and

46 antagonistic behaviour (e.g., defending territory) towards unfamiliar individuals [4].

47 The other factor that affects behaviours towards conspecific individuals is social

48 similarity to the opponent. In such cases, when an animal evaluates an opponent as

49 being a member of the same group (i.e., ingroup), then the animal recognises social

50 similarity to the opponent, regardless of individual familiarity with the opponent. The

51 significance of social similarity and its effect on behaviour have been studied

52 extensively in humans. For example, even people who explicitly endorse egalitarian

53 values are typically not free of biases related to the race or ethnicity of an opponent [5,

54 6]. Nonetheless, relatively few studies have investigated the role that social similarity

55 plays in interactions among non-human animals.

Social buffering refers to the phenomenon in which the presence of affiliative

conspecific individuals mitigates stress responses in a subject [7]. Studies have

demonstrated that, in addition to social buffering by a mother or mate $[8,9]$, social

buffering can be elicited by other conspecific individuals in a variety of species [10-12].

60 We conducted a series of studies in rats to examine whether social buffering is induced

61 by a conspecific individual other than the subject's mother or mate [13, 14]. Stress 
responses caused by an aversively conditioned stimulus were completely blocked when a Wistar subject rat was accompanied by an unfamiliar same-sex Wistar rat, hereafter referred to as the accompanying rat [15-19]. Therefore, social similarity alone appears to be sufficient for inducing social buffering in rats.

In addition to a number of characteristics [20-24], including the importance of olfactory signals [20, 25, 26] and possible neural mechanisms [26-29], social buffering in rats was found to be induced by certain unfamiliar strains of unfamiliar individuals. Specifically, an unfamiliar Sprague-Dawley (SD), Long-Evans (LE), or Lewis accompanying rat had the effect of acting as a social buffer and induced social buffering in the Wistar subject [30]. Conversely, unfamiliar Fischer 344 (F344) or Brown Norway accompanying rats were not regarded as buffers, hereafter referred to as non-buffers, because they did not induce social buffering [30]. Given that the dyad had not met before, individual familiarity with the accompanying rat could not contribute towards social buffering. The remaining possibility might be that the Wistar subject recognised some social similarity to the accompanying rat, even if the subject was unfamiliar with the SD, LE, and Lewis strains. Examining the genealogy of these strains reveals that Lewis rats are derived from Wistar rats, and SD and LE rats are three quarters and half Wistar rats, respectively. Conversely, F344 and Brown Norway rats were established independently of Wistar rats [31]. Therefore, SD, LE and Lewis rats are relatively closely related to Wistar rats, although specific information on actual genetic distances is not currently available. It has been demonstrated that humans will show a preference for an unfamiliar individual if they consider that there is some level of social similarity with that individual [6]. Based on these findings, we hypothesised that a subject would show preference for social buffers over non-buffers. 
87 subjects. Before testing the hypothesis, in Experiment 1, we first examined whether the

88 rats were capable of categorizing unfamiliar rats based on their strain. In a

89 habituation-dishabituation test, the subject was sequentially exposed to three unfamiliar

90 Wistar rats and one unfamiliar F344 rat. In Experiment 2, we examined whether the

91 subject showed a preference for social buffers over non-buffers. We prepared unfamiliar

92 Wistar, SD, and LE rats as social buffers, and unfamiliar F344 rats as non-buffers. In

93 the partner preference test, the subject was offered a choice between a social buffer and

94 non-buffer. The choices between the subject's own strain of social buffer and other unfamiliar strains of social buffer were also offered in order to assess if the subject showed a preference among social buffers. In Experiment 3, we assessed if the subject showed an aversion to non-buffers. The subject was offered a choice between being alone or spending time with a social buffer or non-buffer. In Experiment 4, we assessed whether differences in the behaviour of social buffers and non-buffers contributed to the

100 preference of the subject. The subject was also offered the choice of an anaesthetised

101 social buffer or an anaesthetised non-buffer.

\section{Methods}

All experiments were approved by the Animal Care and Use Committee of the

105 Faculty of Agriculture at The University of Tokyo, according to guidelines adapted

106 from the Consensus Recommendations on Effective Institutional Animal Care and Use

107 Committees by the Scientists Center for Animal Welfare.

109 Animals 
111 (aged 7 weeks), and F344 (aged 9 weeks) rats were purchased from Charles River

112 Laboratories Japan (Kanagawa, Japan). To ensure that body size was similar among rats,

113 we ordered F344 rats of different ages [29, 30]. Upon arrival, all rats were housed

114 individually in a room with an ambient temperature of $24 \pm 1{ }^{\circ} \mathrm{C}$, humidity of $45 \pm 5 \%$,

115 and a 12-h light/12-h dark cycle (lights were switched on at 8:00). Food and water were

116 administered ad libitum. Nylon zip ties (Panduit Japan, Tokyo, Japan) were used as

117 colour bands and attached to the stimulus rats more than $4 \mathrm{~h}$ after arrival, mostly on the

118 following day. All behavioural tests were performed during the light period of the day

119 after the attachment of the colour band.

121 Procedures

122 Experiment 1

The habituation-dishabituation test was performed in an illuminated room

124 using a two-chamber apparatus. The apparatus consisted of two parallel polycarbonate 125 chambers (standard rat cage: $24 \times 40 \times 20 \mathrm{~cm})$. A stainless tube $(6 \mathrm{~cm}$ diameter, $8 \mathrm{~cm}$

126 long) connected the two chambers at approximately one-third the length of the long side

127 of the chambers. One chamber was defined as the starting chamber while the other was

128 defined as the stimulus chamber. The subject was first placed in the starting chamber

129 and allowed to explore the entire apparatus for $10 \mathrm{~min}$. Then, a stimulus rat was tethered

130 in the stimulus chamber to restrict its movement within the chamber. The behaviour of

131 the subject during the subsequent 10-min test period was recorded with a video camera

132 (DCR-TRV18; Sony, Tokyo, Japan) and an HDD-BD recorder (DMR-BW770;

133 Panasonic, Osaka, Japan). After the test period, the subject was returned to the colony 
134 room and kept undisturbed for $10 \mathrm{~min}$. The same procedure was repeated until the

135 subject $(n=6)$ was sequentially presented with three unfamiliar Wistar stimulus rats

136 (individuals A, B, and C) and one unfamiliar F344 stimulus rat (individual D).

138 Experiment 2

139 The partner preference test was performed in an illuminated room using a

140 three-chamber apparatus that was prepared by connecting one identical chamber to the

141 two-chamber apparatus used in Experiment 1. Specifically, three parallel polycarbonate

142 chambers were linearly connected by stainless tubes located at one-third the length of

143 the long side of the chambers. The centre chamber was defined as the starting chamber.

144 The chambers at both ends of the apparatus were defined as the stimulus chambers. The

145 subject was first placed in the starting chamber and allowed to explore the entire

146 apparatus for $20 \mathrm{~min}$. Then, an unfamiliar Wistar, SD, LE, or F344 stimulus rat was

147 tethered individually in each stimulus chamber to restrict its movement within the cage.

148 Each stimulus rat was tethered on opposite sides of the apparatus on consecutive runs.

149 The 60-min test period was started when the subject had visited both stimulus chambers

150 more than once and then returned to the starting chamber. During the test period, the

151 subject was allowed to explore a pair of Wistar and F344 stimulus chambers $(n=7)$, a

152 pair of SD and F344 stimulus chambers $(n=8)$, a pair of LE and F344 stimulus

153 chambers $(n=7)$, a pair of Wistar and SD stimulus chambers $(n=8)$, or a pair of Wistar

154 and LE stimulus chambers $(n=8)$. The behaviour of the subject was recorded with a

155 video camera (DCR-TRV18; Sony, Tokyo, Japan) and an HDD-BD recorder

156 (DMR-BW770; Panasonic, Osaka, Japan). 
160 the exception that only one unfamiliar stimulus rat was used for each subject.

161 Accordingly, the chamber in each end of the apparatus was defined as the empty and

162 stimulus chamber, respectively. During the test period, the subject was allowed to

163 explore a pair of empty and Wistar stimulus chambers $(n=7)$, a pair of empty and SD

164 stimulus chambers $(n=6)$, a pair of empty and LE stimulus chambers $(n=7)$, or a pair 165 of empty and F344 stimulus chambers $(n=6)$. $\mathrm{mg} / \mathrm{kg}$, i.p.). During the test period, the subject was allowed to explore a pair of anaesthetised Wistar and anaesthetised F344 stimulus chambers $(n=8)$.

Data analyses and statistical procedures

A researcher recorded the time spent in each chamber (the centre of the

175 forepaws was considered to indicate the location of the subject) and the time engaged in active social interaction with the stimulus rat (the subject touched the stimulus rat with its nose and/or allo-groomed the stimulus rat). Microsoft Excel-based Visual Basic software was used to record the duration of key presses, as in our previous studies [32, 33]. The data were analysed using the paired $t$ test. The significance level was set at $P<$

$180 \quad 0.05$ for all statistical tests. 
Data are expressed as mean \pm standard error of the means (SEM). We first assessed if the subject could categorise unfamiliar rats based on their

187 strain. In this experiment, the subject was sequentially presented three unfamiliar Wistar

188 stimulus rats (individuals A, B, and C) and one unfamiliar F344 stimulus rat (individual

189 D) in the habituation-dishabituation test (Fig. 1). The time engaged in social interactions

190 with the third Wistar stimulus rat (individual C) was shorter than that with the first

191 Wistar stimulus rat (individual A) $\left(t_{5}=-5.14, P<0.01\right)$. In addition, the time engaged in interactions with the first F344 stimulus rat (individual D) was longer than that with the third Wistar stimulus rat (individual C) $\left(t_{5}=5.47, P<0.01\right)$. In contrast, the time spent in the stimulus chamber did not differ between when the first or third Wistar stimulus rat was presented $\left(t_{5}=-1.89, P=0.12\right)$ (Table 1$)$. Similarly, the time spent in the

196 stimulus chamber did not change when the third Wistar stimulus rat was replaced with

197 the first F344 stimulus rat $\left(t_{5}=1.35, P=0.24\right)$ (Table 1$)$. These results suggested that

198 the subject could categorise unfamiliar rats based on their strain.

Experiment 2

We assessed whether the subject showed a preference for social buffers over non-buffers in the partner preference test (Fig. 2). When a pair of Wistar and F344

203 stimulus rats, a pair of SD and F344 stimulus rats, or a pair of LE and F344 stimulus

204 rats was presented to the subject, the time engaged in social interactions was longer with 205 the Wistar $\left(t_{6}=-3.71, P<0.05\right), \mathrm{SD}\left(t_{7}=-2.43, P<0.05\right)$, or LE stimulus rats $\left(t_{6}=\right.$ 
207 each chamber, the time spent in the Wistar $\left(t_{6}=-3.80, P<0.01\right), \operatorname{SD}\left(t_{7}=-2.47, P<\right.$

208 0.05), and LE stimulus chambers $\left(t_{6}=-3.58, P<0.05\right)$ was longer than that spent in the

209 F344 stimulus chamber (Table 2). These results suggested that the subject showed a

210 preference for social buffers over non-buffers.

211 We also assessed whether the subject showed a preference between social

212 buffers. When a pair of Wistar and SD stimulus rats or a pair of Wistar and LE stimulus

213 rats was presented to the subject rat, the time engaged in social interactions with the

214 Wistar stimulus rat was similar to that with the $\mathrm{SD}\left(t_{7}=0.35, P=0.74\right)$ or LE stimulus

215 rat $\left(t_{7}=-1.14, P=0.29\right)$, respectively (Fig. 2). Similarly, the time spent in the Wistar

216 stimulus chamber was the same as that in the $\mathrm{SD}\left(t_{7}=-1.66, P=0.14\right)$ or LE stimulus

217 chamber $\left(t_{7}=-1.52, P=0.17\right)$ (Table 2$)$. These results suggested that the subject rats did

218 not show a preference among between buffers.

\section{Experiment 3}

Since the partner preference test compares the relative preference intensity of the two stimulus rats, it is unclear whether the subject showed a preference for social buffers or showed aversion to non-buffers. To clarify this point, the subject was offered a choice between being alone and spending time with a social buffer or a non-buffer.

Since there was no stimulus rat in the empty cage, we could not measure the time engaged in social interactions with the stimulus rat in the empty cage. However, we confirmed that the subject interacted with all strains of stimulus rat (Table 4). When we

228 analysed the time spent in each chamber, the time spent in the Wistar $\left(t_{6}=-5.57, P<\right.$ 
$230=-6.73, P<0.01)$ was longer than it was in the empty chamber (Table 3 ). These results

231 suggested that the subject did not show an aversion to non-buffers.

232

233 Experiment 4

It is known that F344 rats are less responsive to social interactions with Wistar rats. For example, co-housing a Wistar rat with an F344 rat is an experimental model of

236 peer-rejection in Wistar rats [34-36]. It is therefore possible that the behaviour of

237 stimulus rats may determine the preference of the subject. To clarify this point, the subject was offered a choice between an anaesthetised social buffer of the same strain as the subject and an anaesthetised non-buffer (Fig. 3). The time engaged in active interaction was longer with the anaesthetised Wistar stimulus rat than with the anaesthetised F344 stimulus rat $\left(t_{7}=-2.48, P<0.05\right)$. However, the time spent in the

242 chamber was similar between the two chambers $\left(t_{7}=0.10, P=0.92\right)$ (Table 4$)$. These

243 results suggested that the subject's preference was less affected by the behaviour of the

244 stimulus rat.

\section{Discussion}

In Experiment 1, the subject showed habituation after the sequential presentation of three unfamiliar Wistar rats. The subject then showed dishabituation when an unfamiliar F344 rat was presented as the fourth individual. These results suggested that the subject could categorise unfamiliar rats based on their strain. In

251 Experiment 2, the subject showed a preference for social buffers over non-buffers. In

252 addition, the subject did not show a preference between social buffers. These results could not be ascribed to the alternative interpretation that the subject showed aversion to 
non-buffers, because the subject showed a preference for non-buffers over being alone differences in behaviour between social buffers and non-buffers, because the subject showed a preference for anaesthetised social buffers over anaesthetised non-buffers in Experiment 4. Based on these results, we concluded that the subject recognised social similarity to certain unfamiliar strains of rats. unfamiliar rats based on their strain. Given the importance of olfactory signals in the recognition of other rats [37], it is possible that the subject recognised a signature mixture of each strain. A signature mixture is a subset of the molecules in an animal's chemical profile that is used to recognise an animal as a member of a particular group $[38,39]$. Body odours, one of the sources of the signature mixture, are determined by genetic factors [40-42]. Because individuals of inbred strains are genetically identical, most components of their body odour are thought to be shared among individuals and to serve as a signature of the inbred strain. Even among individuals of outbred strains, several genetic factors are known to be shared. For example, Wistar rats shared the same allele at 6 out of 27 genetic loci examined among individuals [43]. Therefore, it is reasonable to assume that some components of their body odour are also shared among individuals and that they contribute to the signature mixture of the outbred strain. Taken together, it is possible that the unique odour mixture of each strain enabled the subject to categorise unfamiliar rats based on their strain. 
employed unfamiliar rat strains as opponents in order to assess the role of social

279 similarity in social learning and/or expression of prosocial behaviour [44-46]. However,

280 all of the studies selected the unfamiliar strain based simply on the fact that the subject

281 rat was unfamiliar with the opponent strain. The findings of the present study suggest

282 that the Wistar subject rats classified unfamiliar SD and LE rats as ingroup members.

283 Consistent with this classification is the observation in a previous study in which

284 pairings of unfamiliar SD and LE rats both induced vicarious fear responses in the SD

285 subject [45]. Therefore, it is more appropriate for researchers to select unfamiliar strains

286 of rats based on genealogy or genetic distances, if available. However, in a previous

287 study, the SD subject did not release an unfamiliar LE rat from a restrainer [44]. If the

288 classification suggested in the present study was indeed employed by subject rats, then

289 the SD subject rats should have classified the unfamiliar LE rats as ingroup members

290 and showed helping behaviour. One possible reason for this discrepancy might be the

291 differences in the position of the subject. In the first two studies, the subject was in a

292 position where it could benefit from the opponent, such as receiving buffering effects or

293 danger information. In contrast, the subject had to make an effort to help the restrained

294 rat in the last study. In such a situation, it is reasonable to assume that the subject

295 evaluated the opponent strictly and classified the opponent rats with a narrower range of

296 genetic background as ingroup members. Further analyses are necessary to understand

297 how rats classify opponents as being ingroup members.

298

299 Conclusion

300 In the present study, we demonstrated that the subject showed a preference for

301 social buffers over non-buffers. These results suggest that rats recognise social 
302 similarity to certain unfamiliar strains of rats. The preference observed in the present

303 study shares characteristics with ingroup favouritism in humans. Ingroup favouritism

304 describes the phenomenon in which a person shows a preference for a member of the

305 same group. Race or ethnicity is known to be one of the salient factors that

306 automatically induces such ingroup favouritism [47]. In addition, ingroup favouritism

307 and outgroup derogation are not necessarily reciprocally related [6, 48]. Given that

308 ingroup favouritism based on race and ethnicity emerges in the early stages of life [49],

309 it seems to be a biologically important phenomenon in social animals. Therefore, further

310 analyses on preference among unfamiliar strains of rats may shed light on the

311 neurobiology of sociality in animals.

312

313 Funding

314 This study was supported by JSPS KAKENHI (Grant Numbers 20H03160 and

315 20H04766).

316 


\section{References}

318 [1] Keverne, E. B., Levy, F., Poindron, P., Lindsay, D. R. Vaginal stimulation: an

319 important determinant of maternal bonding in sheep. Science. 219 (1983) 81-3.

320 https://doi.org/10.1126/science.6849123.

321 [2] Williams, J. R., Catania, K. C., Carter, C. S. Development of partner preferences in

322 female prairie voles (Microtus ochrogaster): the role of social and sexual experience.

323 Horm Behav. 26 (1992) 339-49. https://doi.org/10.1016/0018-506x(92)90004-f.

324 [3] Quervel-Chaumette, M., Dale, R., Marshall-Pescini, S., Range, F. Familiarity affects

325 other-regarding preferences in pet dogs. Sci Rep. 5 (2015) 18102.

326 https://doi.org/10.1038/srep18102.

327 [4] Lund, M. Social mechanisms and social structure in rats and mice. Ecol Bull. 19

328 (1975) 255-60.

329 [5] Cunningham, W. A., Preacher, K. J., Banaji, M. R. Implicit attitude measures:

330 consistency, stability, and convergent validity. Psychol Sci. 12 (2001) 163-70.

331 https://doi.org/10.1111/1467-9280.00328.

332 [6] Van Bavel, J. J., Cunningham, W. A. Self-categorization with a novel mixed-race

333 group moderates automatic social and racial biases. Pers Soc Psychol Bull. 35 (2009)

334 321-35. https://doi.org/10.1177/0146167208327743.

335 [7] Kiyokawa, Y., Hennessy, M. B. Comparative studies of social buffering: A

336 consideration of approaches, terminology, and pitfalls. Neurosci Biobehav Rev. 86

337 (2018) 131-41. https://doi.org/10.1016/j.neubiorev.2017.12.005.

338 [8] Hennessy, M. B., Kaiser, S., Sachser, N. Social buffering of the stress response:

339 diversity, mechanisms, and functions. Front Neuroendocrinol. 30 (2009) 470-82.

340 https://doi.org/10.1016/j.yfrne.2009.06.001. 
341 [9] Smith, A. S., Wang, Z. Hypothalamic oxytocin mediates social buffering of the

342 stress response. Biol Psychiatry. 76 (2014) 281-8.

343 https://doi.org/10.1016/j.biopsych.2013.09.017.

344 [10] da Costa, A. P., Leigh, A. E., Man, M. S., Kendrick, K. M. Face pictures reduce

345 behavioural, autonomic, endocrine and neural indices of stress and fear in sheep.

346 Proceedings. Biological sciences / The Royal Society. 271 (2004) 2077-84.

347 https://doi.org/10.1098/rspb.2004.2831.

348 [11] Davitz, J. R., Mason, D. J. Social facilitated reduction of a fear response in rats. J

349 Comp Physiol Psychol. 48 (1955) 149-56. https://doi.org/10.1037/h0046411.

350 [12] Lyons, D. M., Price, E. O., Moberg, G. P. Social modulation of pituitary-adrenal

351 responsiveness and individual differences in behaviour of young domestic goats.

352 Physiol Behav. 43 (1988) 451-8.

353 [13] Kiyokawa, Y. Social Odors: Alarm Pheromones and Social Buffering. Curr Top

354 Behav Neurosci. 30 (2017) 47-65. https://doi.org/10.1007/7854_2015_406.

355 [14] Kiyokawa, Y. Relief provided by conspecifics: social buffering. in: Meyza KZ,

356 Knapska E eds), Neuronal correlates of empathy, Academic Press, 2018.

357 [15] Ishii, A., Kiyokawa, Y., Takeuchi, Y., Mori, Y. Social buffering ameliorates

358 conditioned fear responses in female rats. Horm Behav. 81 (2016) 53-8.

359 https://doi.org/10.1016/j.yhbeh.2016.03.003.

360 [16] Kiyokawa, Y., Hiroshima, S., Takeuchi, Y., Mori, Y. Social buffering reduces male

361 rats' behavioral and corticosterone responses to a conditioned stimulus. Horm Behav. 65

362 (2014) 114-8. https://doi.org/10.1016/j.yhbeh.2013.12.005. 
363 [17] Kiyokawa, Y., Kikusui, T., Takeuchi, Y., Mori, Y. Partner's stress status influences

364 social buffering effects in rats. Behav Neurosci. 118 (2004) 798-804.

365 https://doi.org/10.1037/0735-7044.118.4.798.

366 [18] Kiyokawa, Y., Takeuchi, Y. Social buffering ameliorates conditioned fear

367 responses in the presence of an auditory conditioned stimulus. Physiol Behav. 168

368 (2017) 34-40. https://doi.org/10.1016/j.physbeh.2016.10.020.

369 [19] Kiyokawa, Y., Takeuchi, Y., Mori, Y. Two types of social buffering differentially

370 mitigate conditioned fear responses. Eur J Neurosci. 26 (2007) 3606-13.

371 https://doi.org/10.1111/j.1460-9568.2007.05969.x.

[20] Kiyokawa, Y., Honda, A., Takeuchi, Y., Mori, Y. A familiar conspecific is more effective than an unfamiliar conspecific for social buffering of conditioned fear responses in male rats. Behav Brain Res. 267 (2014) 189-93. https://doi.org/10.1016/j.bbr.2014.03.043.

376 [21] Kiyokawa, Y., Kawai, K., Takeuchi, Y. The benefits of social buffering are

377 maintained regardless of the stress level of the subject rat and enhanced by more

378 conspecifics. Physiol Behav. 194 (2018) 177-83.

379 https://doi.org/10.1016/j.physbeh.2018.05.027.

380 [22] Kiyokawa, Y., Li, Y., Takeuchi, Y. A dyad shows mutual changes during social 381 buffering of conditioned fear responses in male rats. Behav Brain Res. 366 (2019) 45-55.

382 https://doi.org/10.1016/j.bbr.2019.03.024.

383 [23] Mikami, K., Kiyokawa, Y., Ishii, A., Takeuchi, Y. Social buffering enhances 384 extinction of conditioned fear responses by reducing corticosterone levels in male rats.

385 Horm Behav. 118 (2020) 104654. https://doi.org/10.1016/j.yhbeh.2019.104654. 

https://doi.org/10.1016/j.physbeh.2016.05.001. (2009) 777-85. https://doi.org/10.1111/j.1460-9568.2009.06618.x.

392 [26] Takahashi, Y., Kiyokawa, Y., Kodama, Y., Arata, S., Takeuchi, Y., Mori, Y.

393 Olfactory signals mediate social buffering of conditioned fear responses in male rats.

394 Behav Brain Res. 240 (2013) 46-51. https://doi.org/10.1016/j.bbr.2012.11.017. https://doi.org/10.3389/fnins.2015.00099. underlying social buffering of conditioned fear responses in male rats. Eur J Neurosci. 36 (2012) 3429-37. https://doi.org/10.1111/j.1460-9568.2012.08257.x. [29] Minami, S., Kiyokawa, Y., Takeuchi, Y. The lateral intercalated cell mass of the amygdala is activated during social buffering of conditioned fear responses in male rats.

404 Behav Brain Res. 372 (2019) 112065. https://doi.org/10.1016/j.bbr.2019.112065.

405 [30] Nakamura, K., Ishii, A., Kiyokawa, Y., Takeuchi, Y., Mori, Y. The strain of an 
410 [32] Kiyokawa, Y., Kodama, Y., Kubota, T., Takeuchi, Y., Mori, Y. Alarm pheromone

411 is detected by the vomeronasal organ in male rats. Chem Senses. 38 (2013) 661-8.

412 https://doi.org/10.1093/chemse/bjt030.

413 [33] Kobayashi, T., Kiyokawa, Y., Arata, S., Takeuchi, Y., Mori, Y. c-Fos expression

414 during the modulation of sexual behavior by an alarm pheromone. Behav Brain Res.

415237 (2013) 230-7. https://doi.org/10.1016/j.bbr.2012.09.042.

416 [34] Schneider, P., Bindila, L., Schmahl, C., Bohus, M., Meyer-Lindenberg, A., Lutz, B.,

417 et al. Adverse Social Experiences in Adolescent Rats Result in Enduring Effects on

418 Social Competence, Pain Sensitivity and Endocannabinoid Signaling. Front Behav

419 Neurosci. 10 (2016) 203. https://doi.org/10.3389/fnbeh.2016.00203.

420 [35] Schneider, P., Hannusch, C., Schmahl, C., Bohus, M., Spanagel, R., Schneider, M.

421 Adolescent peer-rejection persistently alters pain perception and CB1 receptor

422 expression in female rats. Eur Neuropsychopharmacol. 24 (2014) 290-301.

423 https://doi.org/10.1016/j.euroneuro.2013.04.004.

424 [36] Schneider, P., Patz, M., Spanagel, R., Schneider, M. Adolescent social rejection 425 alters pain processing in a CB1 receptor dependent manner. Eur Neuropsychopharmacol. 42626 (2016) 1201-12. https://doi.org/10.1016/j.euroneuro.2016.04.007.

427 [37] Carr, W. J., Yee, L., Gable, D., Marasco, E. Olfactory recognition of conspecifics 428 by domestic Norway rats. J Comp Physiol Psychol. 90 (1976) 821-8.

429 https://doi.org/10.1037/h0077266.

430 [38] Wyatt, T. D. Pheromones and signature mixtures: defining species-wide signals 431 and variable cues for identity in both invertebrates and vertebrates. J Comp Physiol A

432 Neuroethol Sens Neural Behav Physiol. 196 (2010) 685-700.

433 https://doi.org/10.1007/s00359-010-0564-y. 
434 [39] Wyatt, T. D. Pheromones. Curr Biol. 27 (2017) R739-R43.

435 https://doi.org/10.1016/j.cub.2017.06.039.

436 [40] Boyse, E. A., Beauchamp, G. K., Yamazaki, K. The genetics of body scent. Trends 437 Genet. 3 (1987) 97-102. https://doi.org/10.1016/0168-9525(87)90192-2.

438 [41] Yamazaki, K., Beauchamp, G. K. Genetic basis for MHC-dependent mate choice. 439 Adv Genet. 59 (2007) 129-45. https://doi.org/10.1016/S0065-2660(07)59005-X.

440 [42] Yamazaki, K., Beauchamp, G. K., Matsuzaki, O., Bard, J., Thomas, L., Boyse, E.

441 A. Participation of the murine $\mathrm{X}$ and $\mathrm{Y}$ chromosomes in genetically determined

442 chemosensory identity. Proc Natl Acad Sci U S A. 83 (1986) 4438-40.

443 https://doi.org/10.1073/pnas.83.12.4438.

444 [43] Nakanishi, S., Serikawa, T., Kuramoto, T. Slc:Wistar outbred rats show close 445 genetic similarity with F344 inbred rats. Exp Anim. 64 (2015) 25-9.

446 https://doi.org/10.1538/expanim.14-0051.

447 [44] Ben-Ami Bartal, I., Rodgers, D. A., Bernardez Sarria, M. S., Decety, J., Mason, P. 448 Pro-social behavior in rats is modulated by social experience. Elife. 3 (2014) e01385. 449 https://doi.org/10.7554/eLife.01385.

450 [45] Han, Y., Bruls, R., Soyman, E., Thomas, R. M., Pentaraki, V., Jelinek, N., et al. 451 Bidirectional cingulate-dependent danger information transfer across rats. PLoS Biol. 45217 (2019) e3000524. https://doi.org/10.1371/journal.pbio.3000524.

453 [46] Saggerson, A. L., Honey, R. C. Observational learning of instrumental 454 discriminations in the rat: the role of demonstrator type. Q J Exp Psychol (Hove). 59 455 (2006) 1909-20. https://doi.org/10.1080/17470210600705032. 
456 [47] Amodio, D. M., Devine, P. G. Stereotyping and evaluation in implicit race bias:

457 evidence for independent constructs and unique effects on behavior. J Pers Soc Psychol.

45891 (2006) 652-61. https://doi.org/10.1037/0022-3514.91.4.652.

459 [48] Otten, S., Moskowitz, G. B. Evidence for implicit evaluative in-group bias:

460 Affect-biased spontaneous trait inference in a minimal group paradigm. J Exp Soc

461 Psychol. 36 (2000) 77-89. https://doi.org/10.1006/jesp.1999.1399.

462 [49] Xiao, N. G., Quinn, P. C., Liu, S., Ge, L., Pascalis, O., Lee, K. Older but not

463 younger infants associate own-race faces with happy music and other-race faces with

464 sad music. Dev Sci. 21 (2018). https://doi.org/10.1111/desc.12537.

465 
466 Table 1. Time spent in the stimulus chamber in Experiment 1

\begin{tabular}{lr}
\hline Stimulus rat & Time spent in the ch \\
\hline Wistar (individual A) & $509 \pm 17$ \\
Wistar (individual B) & $429 \pm 18$ \\
Wistar (individual C) & $448 \pm 30$ \\
F344 (individual D) & $470 \pm 30$
\end{tabular}

467 Data are expressed as mean \pm standard error of the mean.

468 F344: Fischer 344.

469 
470 Table 2. Time spent in the stimulus chamber in Experiment 2

Stimulus rat

Time spent in the chamber (min)

Wistar vs. F344

Wistar

$28.3 \pm 2.3^{*}$

F344

$13.8 \pm 2.1$

SD vs. F344

SD

$27.5 \pm 4.0 *$

F344

$14.0 \pm 2.1$

LE vs. F344

LE

$23.3 \pm 3.2 *$

F344

$8.8 \pm 1.7$

Wistar vs. SD

Wistar

$24.4 \pm 3.6$

SD

$17.0 \pm 2.5$

Wistar vs. LE

Wistar

$28.4 \pm 5.3$

LE

$13.8 \pm 4.7$

471 Data are expressed as mean \pm standard error of the mean.

472 F344: Fischer 344; LE: Long-Evans; SD: Sprague-Dawley.

$473 * P<0.05$ for paired $t$-test.

474 
475 Table 3. Results of Experiment 3

Stimulus rat Time spent in the chamber Time engaged in interaction

(min)

Wistar vs. Empty

Wistar

$$
32.4 \pm 3.6^{*}
$$

$6.8 \pm 1.4$

$$
453 \pm 78
$$

Empty

$$
34.6 \pm 4.5^{*}
$$

$141 \pm 23$

Empty

$6.1 \pm 0.9$

N/A

LE vs. Empty

LE

$33.0 \pm 5.0 *$

$213 \pm 34$

Empty

$7.2 \pm 2.5$

N/A

F344 vs. Empty

F344

$37.0 \pm 4.3^{*}$

$93.3 \pm 12.5$

Empty

$4.2 \pm 0.7$

N/A

476 Data are expressed as mean \pm standard error of the mean.

477 F344: Fischer 344; LE: Long-Evans; N/A: not applicable; SD: Sprague-Dawley.

$478 * P<0.05$ for paired $t$-test.

479 
480 Table 4. Time spent in the stimulus chamber in Experiment 4

Stimulus rat

Time spent in the chamber ( $\min )$

Anaesthetised Wistar

$11.7 \pm 5.8$

Anaesthetised F344

$12.5 \pm 5.1$

481 Data are expressed as mean \pm standard error of the mean.

482 F344: Fischer 344.

483 


\section{$484 \quad$ Figure captions}

485 Fig. 1. Time the subject $(n=6)$ engaged in social interaction with three unfamiliar,

486 sequentially presented, Wistar stimulus rats (individuals A, B, and C) and one

487 unfamiliar Fischer 344 (F344) stimulus rat (individual D) in Experiment 1. $* P<0.05$

488 with paired $t$-test.

489

490 Fig. 2. Time the Wistar subject $\left(\mathrm{W}_{\mathrm{S}}\right)$ engaged in social interaction with the stimulus rats

491 when allowed to explore a pair of Wistar (W) and Fischer $344(\mathrm{~F})$ stimulus chambers ( $n$

$492=7)$, a pair of Sprague-Dawley (SD) and F stimulus chambers $(n=8)$, a pair of

493 Long-Evans (LE) and F stimulus chambers $(n=7)$, a pair of $\mathrm{W}$ and SD stimulus

494 chambers $(n=8)$, or a pair of $\mathrm{W}$ and LE stimulus chambers $(n=8)$ in Experiment 2. *P

$495<0.05$ with paired $t$-test.

496

497 Fig. 3. Time the Wistar subject $\left(\mathrm{W}_{\mathrm{s}}\right)$ engaged in social interaction with the stimulus rats

498 when allowed to explore a pair of anaesthetised Wistar $\left({ }^{\mathrm{a}} \mathrm{W}\right)$ and anaesthetised Fischer

$499344\left({ }^{\mathrm{a}} \mathrm{F}\right)$ stimulus chambers $(n=8)$ in Experiment $4 . * P<0.05$ with paired $t$-test.

500

501

502

503

504

505

506

507 


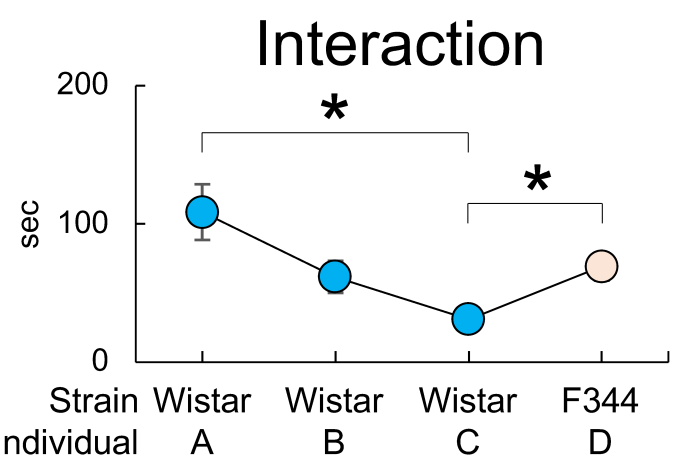



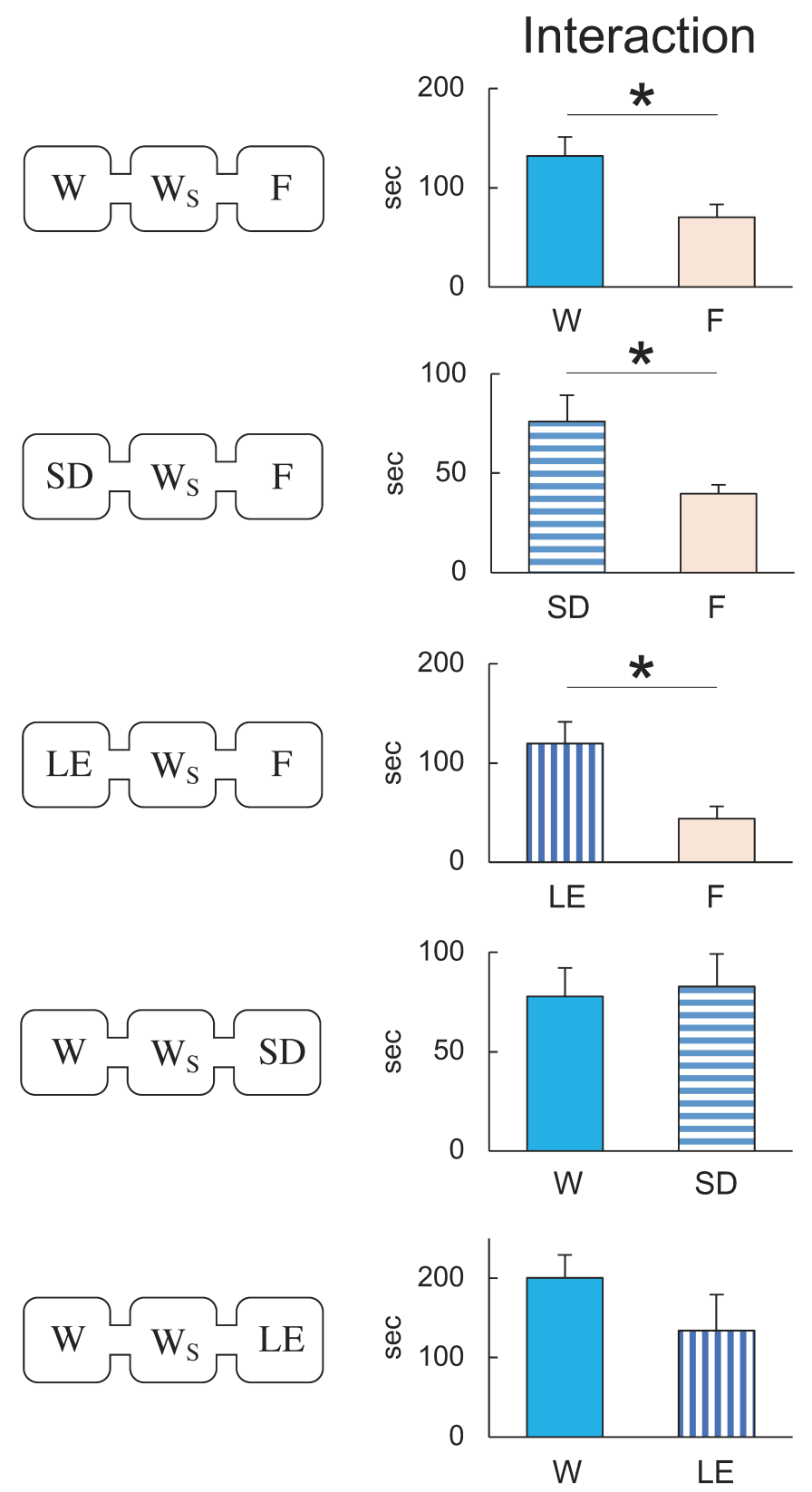

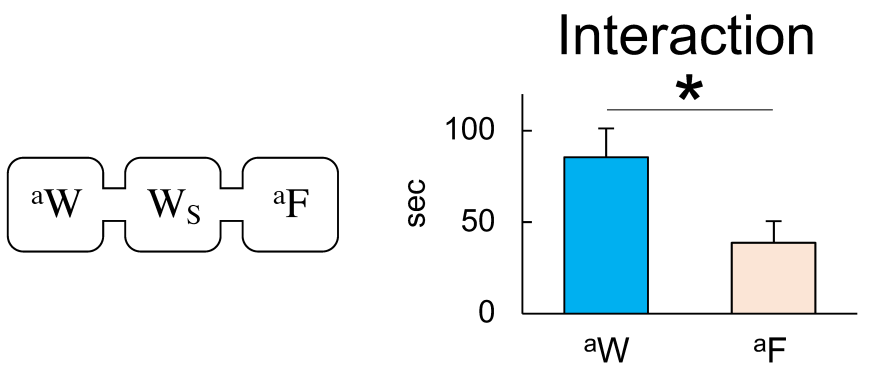